\title{
Field Experiments in Mobility and Navigation with a Lunar Rover Prototype
}

\author{
David Wettergreen ${ }^{1}$, Dominic Jonak, David Kohanbash, Scott Moreland, \\ Spencer Spiker, and James Teza
}

\begin{abstract}
Scarab is a prototype rover for lunar missions to survey resources, particularly water ice, in polar craters. It is designed as a prospector that would use a deep coring drill and apply soil analysis instruments. Its chassis can transform to stabilize its drill in contact with the ground and can also adjust posture to ascend and descent steep slopes. Scarab has undergone field testing at lunar analogue sites in Washington and Hawaii in an effort to quantify and validate its mobility and navigation capabilities. We report on results of experiments in slope ascent and descent and in autonomous kilometer-distance navigation in darkness.
\end{abstract}

\section{Introduction}

To discover and measure the resources of the moon, robotic systems will have to survive extremes from blazing sunlight to frigid darkness as well as dust, vacuum, and isolation. Scarab is a prospecting rover developed to perform the necessary science operations to locate volatiles and validate in situ resource utilization methods. [Sanders09] (Fig. 1) It is a terrestrial concept vehicle designed to deploy a deep coring drill and to transport

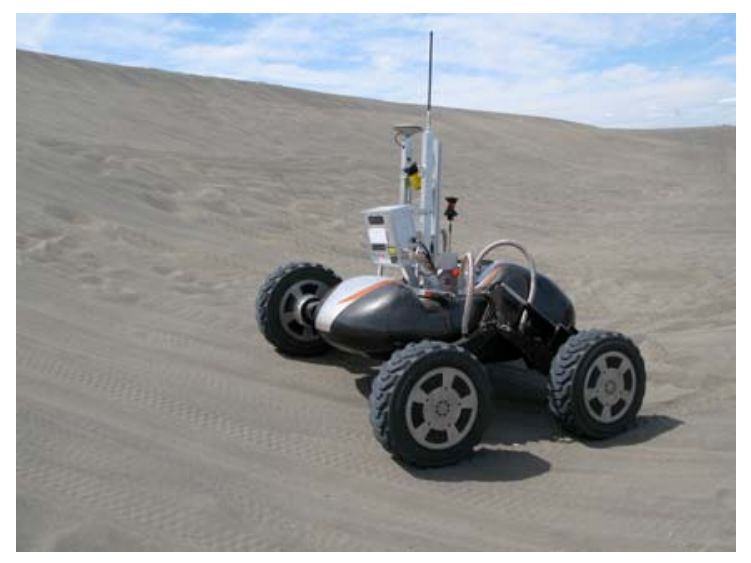

Figure 1. Scarab lunar rover prototype on unconsolidated sandy soil in eastern Washington soil analysis instruments. The vehicle design employs a passive kinematic suspension with active posture adjustability. Its chassis can lower to stabilize a coring drill in contact with the ground and can also adjust to control roll, meaning

\footnotetext{
${ }^{1}$ Carnegie Mellon University, 5000 Forbes Avenue.Pittsburgh, PA 15213, dsw@ri.cmu.edu
} 
rotation about its longitudinal axis, by independently adjusting its side-frames. This allows it to drive cross-slope and turn switchbacks to better ascend and descend unconsolidated soil.

Scarab is intended to operate on and within lunar craters, particularly in polar regions. Because the interior slopes and crater floor are sometimes in shadow, or in some cases in permanent darkness, active sensing methods are needed for terrain modeling and autonomous navigation. Scarab employs laser range scanners with autonomous navigation algorithms to build models of the surrounding terrain to detect obstacles and then determine efficient and safe paths.

In this paper we review results from field experiments at Moses Lake Dunes, Washington and Mauna Kea, Hawaii to measure and verify the prototype rover's ability to meet the demands of a lunar polar crater prospecting mission.

\section{Rover Configuration}

Scarab was conceived as a work machine with a serialized mission: drive, charge batteries, drill, charge again, analyze soil samples, charge and repeat. The number of repetitions might be 25 , leading to 25 kilometers of traverse, 25 cores, and 25 sites surveyed. For some craters, 100 repetitions might be more desirable to characterize the environment and resources.

There are many factors effecting the rover configuration but the drill mechanism and its operation dominate. The requirement to transport and stabilize a deep coring drill literally became central to the design while requirements for ascent and descent in cratered terrain shaped many aspects and fine details.

Drilling requires a stiff platform into which thrust loads, torques and vibrations are transmitted and hole alignment is maintained. Placement of the drill in line with the vehicle's center-of-mass maximizes the mass that can be applied in down force. (Fig. 2) Drilling operations receive three benefits of this feature; first, lowering the chassis allows the full stroke of the drill to be used in the soil resulting in mass savings overall. Secondly, the rover can lean and therefore re-stabilize and place the rover center-of-mass over the drill core. Lastly, under low gravity conditions, the drill torques are counteracted by the increased leverage arm created by spreading the rover wheelbase.

The rationale for the vehicle weight and size is based on the $1 \mathrm{~m}$ long, $3 \mathrm{~cm}$ diameter drill that is likely to be employed in a lunar mission. Not only does the rover have to support the drill but also it must provide sufficient weight against which the drill can react its downward thrust and torque about the bit. Drill thrusts are expected to reach $250 \mathrm{~N}$ and $50 \mathrm{Nm}$ torque. The system weight on lunar surface must react drilling $250 \mathrm{~N}$ downforce and maintain $150 \mathrm{~N}$ on wheels for stability against uplift and spin, therefore total weight on lunar surface must be greater than $400 \mathrm{~N}$. The weight in lunar gravity $\left(400 \mathrm{~N} / 1.622 \mathrm{~m} / \mathrm{s}^{2}=250 \mathrm{~kg}\right)$ leads to a minimum $250 \mathrm{~kg}$ vehicle mass. [Bartlett08] 
The Scarab rover's chassis and suspension was designed around the drill. This component of the payload is significant in mass $(50 \mathrm{~kg})$ and imposes forces on the chassis during transport and while interacting with the ground.

Scarab's chassis allows it to passively conform to the terrain. The suspension has active and passive elements for improved traction on slope terrain. The active element, as previously discussed with respect to drilling, allows the rover to level the body, leading to increased stability and traction efficiency. The passive element, sometimes called an averaging (or differencing) linkage provides a mechanical release allowing the two rover suspension side-frames to pivot independently.

The averaging linkage ensures the body is pitchaveraged between the rocker arms. Scarab's body has three contact points. On either side, the body is connected to the pivot in the rocker arms. On top, the body hangs from the differencing linkage. This linkage runs across the top of the body and also connects to the rocker arms. Scarab actively controls its roll using the rocker arms by changing the height of each

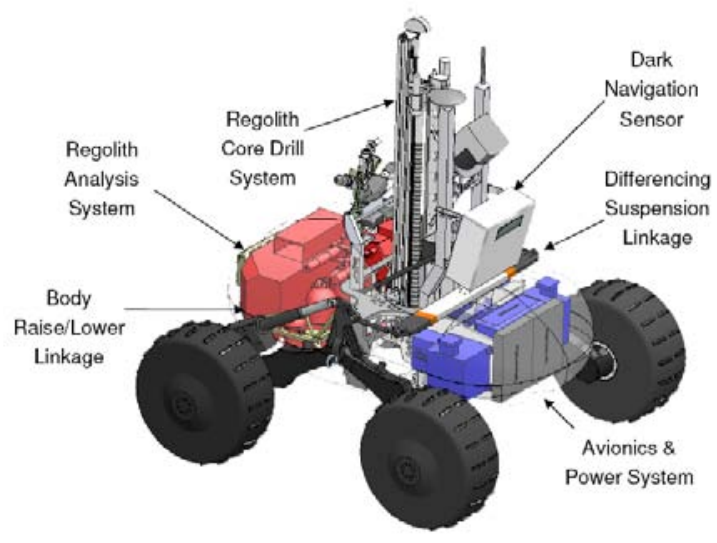

Figure 2. Scarab rover configuration showing placement of sensors, avionics, and payload. There are drive motors in each of four wheels and two linkages for adjusting sideframe height. An averaging linkage allows all four wheels to maintain ground contact in rough terrain.

\begin{tabular}{|c|c|}
\hline Mass & $280 \mathrm{~kg}$ \\
\hline \multirow[t]{2}{*}{ Weight } & 2740 N Earth surface \\
\hline & 450 N Lunar surface \\
\hline Locomotion speed & $3-6 \mathrm{~cm} / \mathrm{s}$ \\
\hline Wheel diameter & $65 \mathrm{~cm}$ \\
\hline Track width & $1.4 \mathrm{~m}$ \\
\hline \multirow[t]{2}{*}{ Wheelbase } & $0.8-1.4 \mathrm{~m}$ \\
\hline & $1.2 \mathrm{~m}$ nominal \\
\hline \multirow{3}{*}{$\begin{array}{l}\text { Aspect ratio } \\
\text { (track/wheelbase) }\end{array}$} & 1:1.0 low stance \\
\hline & 1:1.2 nominal stance \\
\hline & 1:1.7 high stance \\
\hline CG planar location & On geometric center \\
\hline \multirow[t]{3}{*}{ CG height } & $0.48 \mathrm{~m}$ low stance \\
\hline & $0.64 \mathrm{~m}$ nominal stance \\
\hline & $0.74 \mathrm{~m}$ high stance \\
\hline \multirow[t]{3}{*}{ Static pitch-over } & $56^{\circ}$ low stance \\
\hline & $43^{\circ}$ nominal stance \\
\hline & $30^{\circ}$ high stance \\
\hline \multirow[t]{3}{*}{ Static roll-over } & $61^{\circ}$ low stance \\
\hline & $53^{\circ}$ nominal stance \\
\hline & $49^{\circ}$ high stance \\
\hline Maximum straddle & $0.55 \mathrm{~m}$ \\
\hline Minimum straddle & $0.00 \mathrm{~m}$ (ground contact) \\
\hline
\end{tabular}


side independently, thus controlling the roll. In contrast the pitch is passive. Scarab's wheels conform to the terrain, which rotates the rocker arms and swivels the differencing linkage. The linkage is constructed such that the body is forced to move up or down by half the angle between the two rocker arms. As the center-ofmass of the rover is located midway between the side frames, equal loading occurs on all four wheels even on drastically uneven terrain.

\section{Mobility Experiments}

Testing Scarab in the field has been critical in proving the concept for lunar mobility and quantifying performance. Experiments have been conducted in numerous conditions with several findings of importance. However it is understood that continuing experimentation is needed to provide the data for a fully validated performance model and, most important, to enable extrapolation of terrestrial results to the lunar environment.

Moses Lake Sand Dunes in Washington was chosen as a test site for its varied terrain (slopes, pits, etc.), low moisture content, varied soil types (strengths, size distribution) and wide open space. These qualities provided grounds for mobility traction testing and long distance dark navigation traverses. Steep slope ascent/ descent in loose soil and tests of new slope climbing techniques and algorithms were the focus of these field tests in June 2008.

Another lunar analogue site, located on Mauna Kea, Hawaii, is at high altitude with dry, deep, basaltic volcanic ash allows repeated mobility and navigation experiments. The soil composition and mechanical properties at this site were ideal for the regolith sampling hardware experiments. The objectives of these tests in November 2008 were to demonstrate roving, drilling, sample acquisition, processing and analysis. The rover was able to autonomously traverse kilometers of rough terrain, inspect a drill site, drill to $1 \mathrm{~m}$ depth, process the core samples and analyze the composition of the captured soil and demonstrate extraction of water from soil.

Characterization of Scarab as a system for difficult terrain mobility was first quantified in the laboratory in statics tests and in sandboxes. [Bartlett08] The independently actuated rocker arms of the Scarab rover allow for actively controlled center-of-mass shifting. The JPL Sample Return Rover has similar capability [Iagnemma00]. Benefits of this feature include decreased slip during cross-slope maneuvers. Scarab was tested normal to the slope and leaning to maintain vertical posture with cross-slope of $10^{\circ}, 15^{\circ}$ and $20^{\circ}$. A surveying total station tracked a prism on the rover to millimeter accuracy and

Table 2. Downhill Slip

Slope Normal Leaning Change

$10^{\circ} \quad 6 \% \quad 2 \% \quad-4 \%$

$15^{\circ} \quad 22 \% \quad 8 \% \quad-14 \%$

$20^{\circ} \quad 37 \% \quad 15 \% \quad-22 \%$
recorded instantaneous slip measurements. The outcome, expressed as percentage downhill slip with respect to cross-slope distance, appears in Table 2.

The considerable decrease in downhill slip $(2.5 \mathrm{x}$ 
at $20^{\circ}$ incline) arises from increased traction due to equalization of wheel loading in highly cohesive soils and edging effects of the wheel profile in frictional soils. The significance of this outcome lies in the ability to descend and navigate steeper slopes with while maintaining adequate control authority.

A widely used metric for measuring the total tractive ability of a vehicle is drawbar pull. This is the value the vehicle can pull in a specified material while maintaining forward progress. The maximum drawbar pull value corresponds to the inflection in the load/slip curve where the soil fails and the wheel enters the high slip regime. This value is informative when comparing different designs and also used for determining the slope a vehicle can ascend for the specified material. Drawbar pull experiments were conducted in Washington and Hawaii to evaluate of the effects of rover mass properties, wheel design, and soil properties on tractive performance. (Fig. 3) Both the drawbar pull value and power values derived from this test are used as metrics to determine performance.

The key observations are the range of tractive values that occur with changing soil properties. For high bearing strength materials, the level of looseness and compaction does result in slightly varied traction and power (shear strength and sinkage respectively). The overall mass also has little effect on the normalized drawbar pull value (percentage of vehicle weight) although with extremely low bearing strength materials, this does not hold true as a result of excessive sinkage. The shear strength comes from cohesiveness and internal friction. As a result, the drawbar pull values can be representative of slopes achievable for only highly cohesive material as the normal loading of the surface is constant throughout testing. The most significant effect on traction has resulted in wheel design. Experiments involving different traction surfaces, wheel diameter and ground pressures have shown a large range of drawbar pull values. Differences of $50 \%$ have been achievable through traction surface/grouser modifications. Lowering ground pressure and reducing sinkage has moderate effects on traction but results in large differences in driving power (up to $50 \%$ during experiments). Drawbar pull tests per-

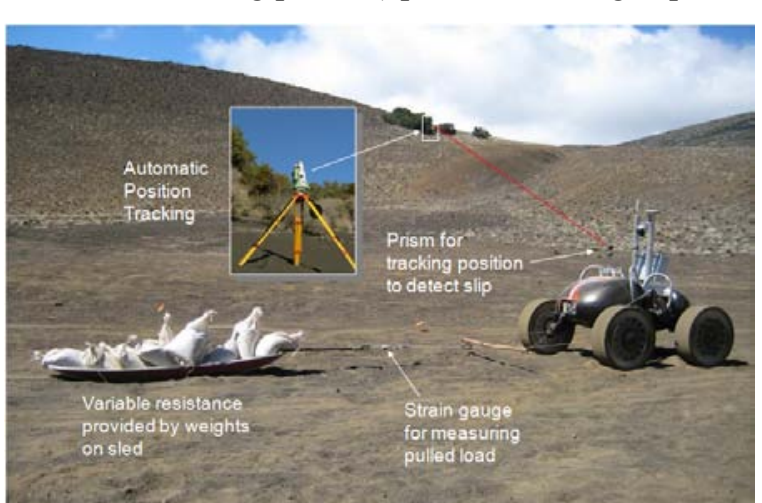

Figure 3. Drawbar pull experimental setup. Weight is added to the sled with the rover in motion while slip is continuously monitored. formed as lab and field experiments have highlighted wheel design as a leading element in tractive and power design requirements. (Table 3.) This is important because wheel design is generally independent of the suspension design and can be optimized for traction and power efficiencies.

Active control methods can also lead to increased tractive performance. 
Techniques such as "inchworming" can increase the mobility of a rover. (Fig. 4) To begin the cycle of inchworming, the body lowers while expanding the wheelbase and rolling the front wheels forward while the rear wheels remains static. In the second half of the cycle, the body raises and the wheel base shortens while the rear wheels rolls forward and the front wheels remains static. Non-rotating wheels provide a fixed point of reaction with no

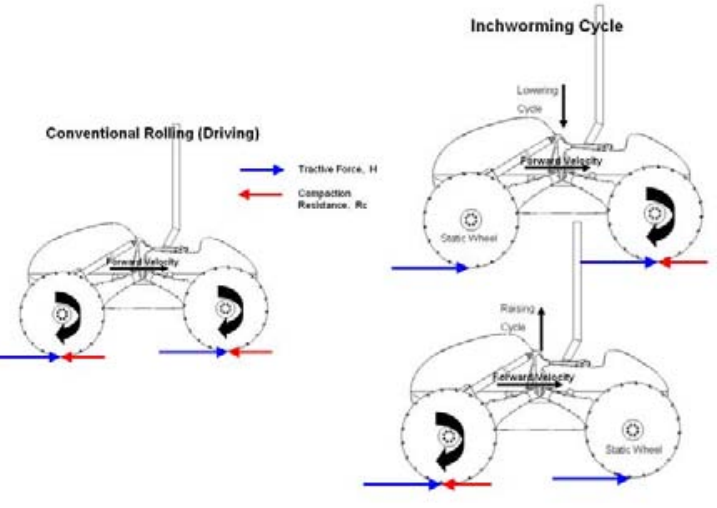

Figure 4. Conventional rolling versus inchworming where one wheel pair is synchronized to the side-arm expansion/contraction and the other reacts forces into the ground. slipping. To achieve these benefits, Scarab's inch-worming algorithm relies on eliminating the compaction resistances on two of the four wheels, by remaining static with respect to ground, for a resulting net tractive increase. Experimentally we have found that the inchworming technique is best suited when wheels become entrenched under high slip. It allows the rover to move forward (or back up) out of this situation.

Actively-positioned center-of-mass can also increase steepness of slopes traversable: distributing the load amongst the rover's wheels leads to more efficient traction. Center-of-mass shifting (body leaning) was tested and heading slip, the slip in the commanded direction with respect to the commanded velocity, showed increase the steepness of slopes ascendable. The experiments were conducted with a $25^{\circ}$ angle attack from the horizontal. This value was determined experimentally to have adequate uphill progress and low slip. It was shown that with the transformable suspension of the Scarab rover, slopes of $20^{\circ}$ loose, dry, volcanic ash can be ascended with low risk.

\begin{tabular}{l|l|l|l|l}
\hline Table 3. & Soil Depth & Lunar Wheel & Rubber Tire & Difference \\
\hline \multirow{2}{*}{ Locomotion Power } & $7.5 \mathrm{~cm}$ & $100 \mathrm{~W}, 100 \mathrm{~W}$ w/grouser & $158 \mathrm{~W}$ & 0.58 \\
\cline { 2 - 5 } & $5.0 \mathrm{~cm}$ & $95 \mathrm{~W}$ & $160 \mathrm{~W}$ & 0.68 \\
\cline { 2 - 5 } & $2.5 \mathrm{~cm}$ & $95 \mathrm{~W}$ & $103 \mathrm{~W}$ & 0.08 \\
\cline { 2 - 5 } & $1 \mathrm{~cm}$ & $98 \mathrm{~W}$ & $117 \mathrm{~W}$ & 0.19 \\
\hline $\begin{array}{l}\text { Maximum Drawbar } \\
\text { Pull }\end{array}$ & $7.5 \mathrm{~cm}$ & $23 \%, 32 \%$ w/grouser & $28 \%$ & $0.18,0.28$ \\
\cline { 2 - 5 } & $5.0 \mathrm{~cm}$ & $24 \%$ & $32 \%$ & 0.25 \\
\cline { 2 - 5 } & $2.5 \mathrm{~cm}$ & $32 \%$ & $39 \%$ & 0.18 \\
\cline { 2 - 5 } & $1 \mathrm{~cm}$ & $33 \%$ & $50 \%$ & 0.34 \\
\hline
\end{tabular}




\section{Navigation Experiments}

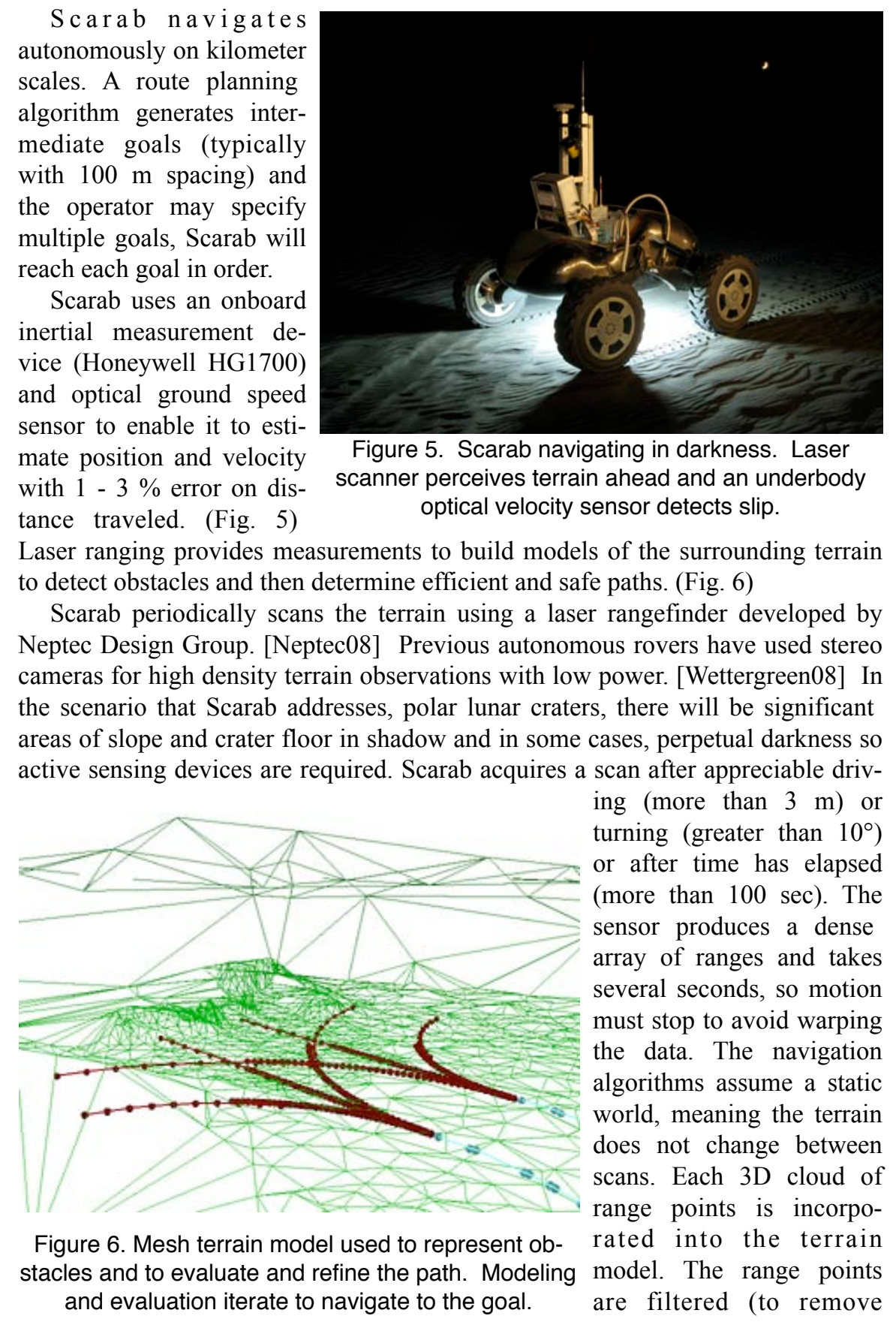


noise and artifacts) and transformed into the vehicle's coordinate frame. Coarse data reduction on the point cloud is applied and the point cloud is transformed into a mesh. The mesh is then further reduced to eliminate redundant data. Finally the mesh is aligned with prior data to generate the terrain model that is used to identify obstacles and select the best path to the goal. Many candidate vehicle motions are evaluated in the near- and far-field. The near-field analysis involves simulating vehicle motion on the mesh to identify collision and slope hazards and assess their severity. The far-field analysis applies heuristic search to estimate the progress each potential move will make toward the goal. A cost function combines safety in the near-field with progress in the farfield.

Our experimental approach has been to conduct $1 \mathrm{~km}$ traverses in a variety of terrains with progressive improvements to algorithms. At both the Moses Lake and Mauna Kea sites, Scarab autonomously completed the following objectives: travel over 3 $\mathrm{km}$, perform 2 night traverses and simulate crater
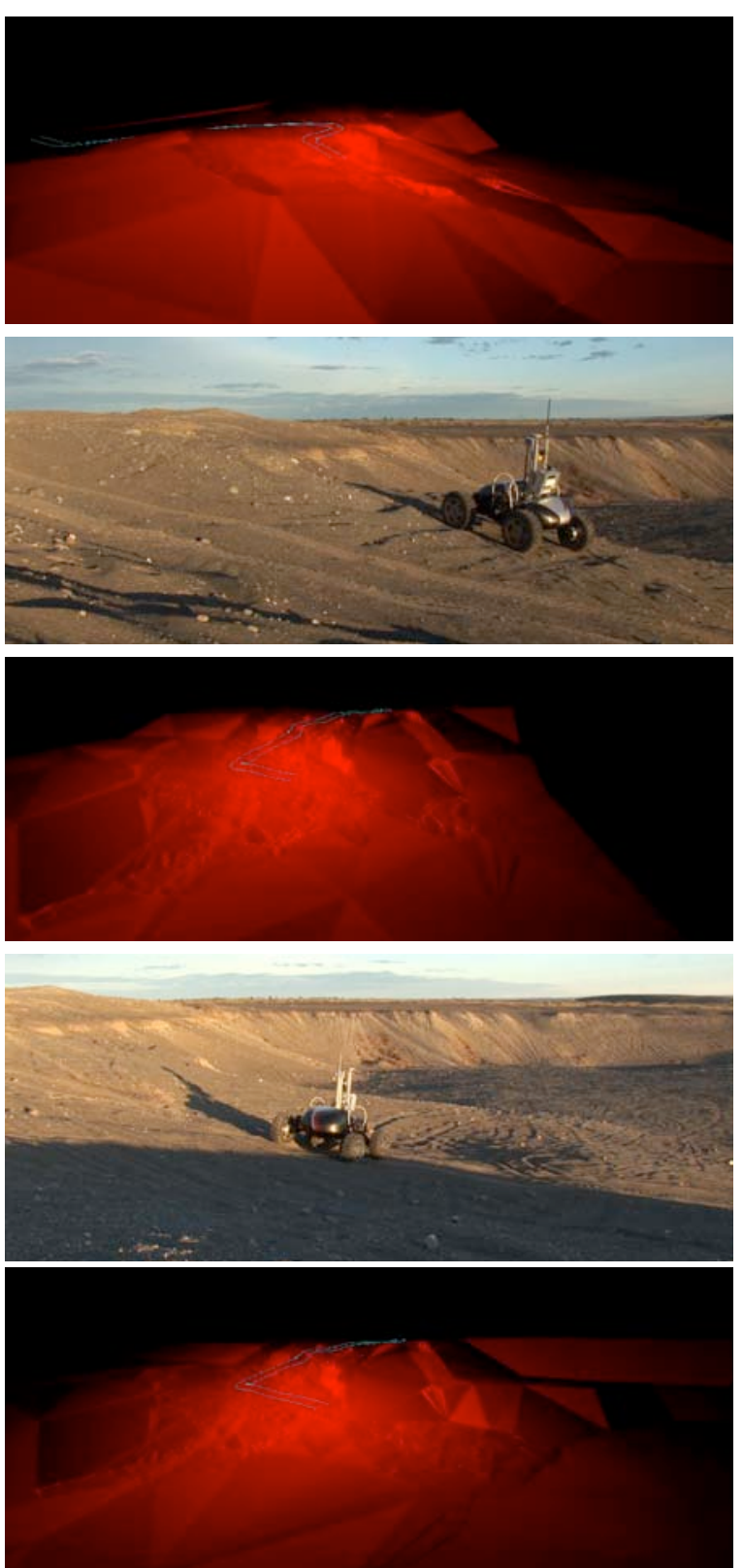

Figure 7. Autonomous descent into a large pit. Rendered views are terrain model with path of rover at rim, intermediate, and viewing the floor. Images show rover during descent. Scarab discovered a moderate slope and reached the floor autonomously. 
descent. Traverses are kilometer scale and performed after sunset, they account for most of the total distance traveled at each site. Crater descent was conducted with a long $(100 \mathrm{~m})$ traverse that included descending a steep $\left(10^{\circ}\right)$ slope.

Scarab completed a total of $3.6 \mathrm{~km}$ in 27 traverses in Washington. The first dark traverse was $1.2 \mathrm{~km}$ with 4 interventions due to sensor faults and one due to a controller error. These faults are recoverable; they do not jeopardize the rover and are easily resolved by resetting a device. A second dark traverse used an alternative navigation algorithm [Pedersen08] and completed $1.1 \mathrm{~km}$ with two interventions due to localization errors.

Scarab traveled $3.0 \mathrm{~km}$ in 20 traverses at Mauna Kea, most of this was accomplished during the two overnight traverses. The first dark traverse was split into two parts; after $199 \mathrm{~m}$ the traverse was paused for logistic reasons and later Scarab resumed for an additional $779 \mathrm{~m}$ before stopping due to a software error. The second overnight traverse was also split in two; the first part was $312 \mathrm{~m}$ and stopped on a software error, the second was $989 \mathrm{~m}$ and ended with a CANBus fault. All of these errors are recoverable remotely.

At each site, Scarab autonomously completed a simulated crater descent using available analogue terrain. At Moses Lake, Scarab drove into a $9 \mathrm{~m}$ deep pit with $10^{\circ}-20^{\circ}$ sloped sides. (Fig. 7) This was safely accomplished including two undirected switchback maneuvers. At Mauna Kea, Scarab repeatedly drove down a winding drainage channel. The route was over $100 \mathrm{~m}$ long and descended over 25 $\mathrm{m}$ with an average grade of about $10^{\circ}$.

Traverse termination conditions for both field tests are shown in Chart 1. No interventions were required to stop the vehicle from driving into a hazard (zero emergency stops). At Moses Lake, most traverses (15 of 25) ended with a recoverable fault. On Mauna Kea the navigation method had improved and most traverses ended by reaching the goal ( 8 of 20) or stopping the traverse for other reasons (6 of 20). Recoverable faults are those that could be remotely corrected and thus would not be mission ending in a lunar scenario.

These results are far from perfect but indications are that reliability is

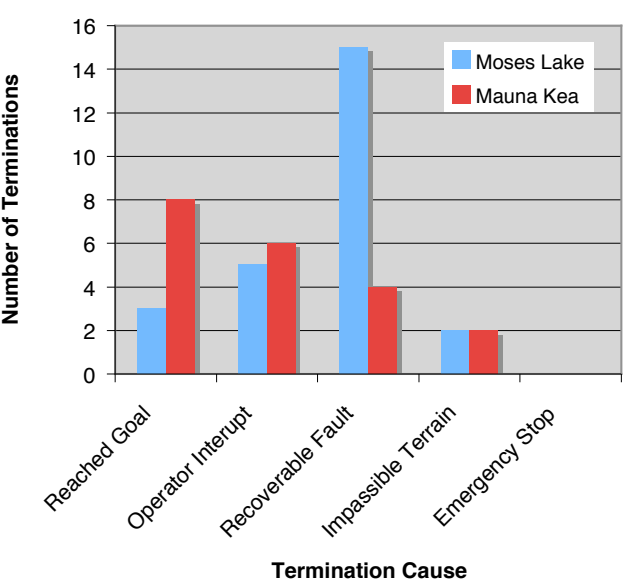

Chart 1. Termination conditions in autonomous navigation experiments. previous planetary rover prototypes. 


\section{Conclusion}

The Scarab rover has been uniquely configured for the transport and stabilization of a coring drill and associated soil analysis instruments. The benefits of central-mounting and active body height and roll control are apparent in deployment of the drill and improved ability to ascend and descend cross-slope.

Field experimentation has quantified drawbar pull and slope climbing ability as well as power required for these activities under a variety of soil and terrain conditions. Field demonstrations have also proven the capability of the laser-based navigation system for kilometer-scale autonomous traverse, including autonomous descent into a crater. In total the mobility and navigation requirements for a lunar surface prospecting mission have been demonstrated in analogue terrain.

\section{Acknowledgments}

We gratefully acknowledge the contributions of our technical team and the assistance of Phillipe Ayoub, Paul Bartlett, Deborah Sigel, John Thornton, Chuck Whittaker, and William Whittaker. This research was supported by NASA under grants, NNX07AE30G, John Caruso, Project Manager, and NNX08AJ99G, Robert Ambrose, Program Scientist.

\section{References}

[Bartlett08] Bartlett, P., Wettergreen, D., and Whittaker, W., "Design of the Scarab Rover for Mobility and Drilling in Lunar Cold Traps," International Symposium on Artificial Intelligence, Robotics and Automation in Space (iSAIRAS), Los Angeles, February 2008.

[Iagnemma00] Iagnemma, K., Rzepniewski, A., Dubowsky, S. et al., "Mobile robot kinematic reconfigurability for rough-terrain," SPIE 2000.

[Neptec08] http://www.neptec.com/Neptec_TriDAR.html

[Pedersen08] Pedersen, L., et al., "Dark Navigation: Sensing and Rover Navigation in Permanently Shadowed Lunar Craters," iSAIRAS, Los Angeles, February 2008.

[Sanders09], Sanders, G., et al., "In Situ Resource Utilization (ISRU) Program," AIAA Aerospace Sciences, Orlando, January 2009.

[Spudis06] Spudis, P., “Ice on the Moon”, The Space Review, November 2006.

[Wettergreen08], Wettergreen, D., et al., "Long-distance Autonomous Survey and Mapping in Robotic Investigation of Life in the Atacama," iSAIRAS, Los Angeles, February 2008.

[Wettergreen09], Wettergreen, D., et al., "Design and Experimentation of a Rover Concept for Lunar Crater Resource Survey,” AIAA Aerospace Sciences, Orlando, January 2009. 